\section{Lateral Sub-cutaneous Route for Anterior Tibial Artery Revascularisation}

Sir,

Below the knee revascularisations using venous grafts decrease the risk of major amputations. ${ }^{1}$ Traditionally, anterior tibial artery (ATA) is revascularised via medial route. In this graft is routed through adductor canal, then tunnelled posterior to knee between the femoral condyle and brought from medial incision to anterior compartment via a tunnel through the interosseus membrane. Lateral route is unconventional route to the ATA. It is recommended in case of scarred or infected groin.2,3 The conventional route is technically difficult, associated with higher risk of graft kinking and is difficult to monitor. ${ }^{4}$

We report a case of successful ATA bypass using lateral subcutaneous route.

A 64-year woman was having right $4^{\text {th }}$ toe gangrene and bilateral rest pain. She was bed bound for the last 4 months due to this condition. Computed angiography showed diffusely diseased superficial femoral and popliteal arteries with the ATA as the only outflow artery in the leg. Angioplasty was unsuccessful because of the long lesion. She was considered for femoro-distal bypass. At presentation, she had severe groin fungal infection and was treated with antifungal medications. Patient had good volume, palpable, femoral arteries. Using profunda femoris artery as inflow, bypass was performed initially for the right leg with tissue loss. Profuda femoris artery and ATA were exposed through standard longitudinal incisions. Ipsilateral great saphenous vein (GSV) was harvested through skip incisions technique, avoiding infected areas and used in reverse fashion. Lateral subcutaneous route was created using a $36 \mathrm{~F}$ chest tube. A counter incision was required in the upper third thigh. She had moderately diseased, calcified ATA. Right common femoral and profunda femoris arteries were having minimal plaque and good pulsatile flow. End-to-side anastomoses of the graft were created with profunda femoris and ATA and inline flow established up to the foot. Wounds were closed in layers. Patient had smooth recovery and was discharged on fifth postoperative day. The gangrenous $4^{\text {th }}$ toe auto-amputated at third week.

After 4 weeks of first operation, she underwent revascularisation using same approach on the left side. A small abscess on the inner upper thigh noted at the time of operation was drained, covered and separated from

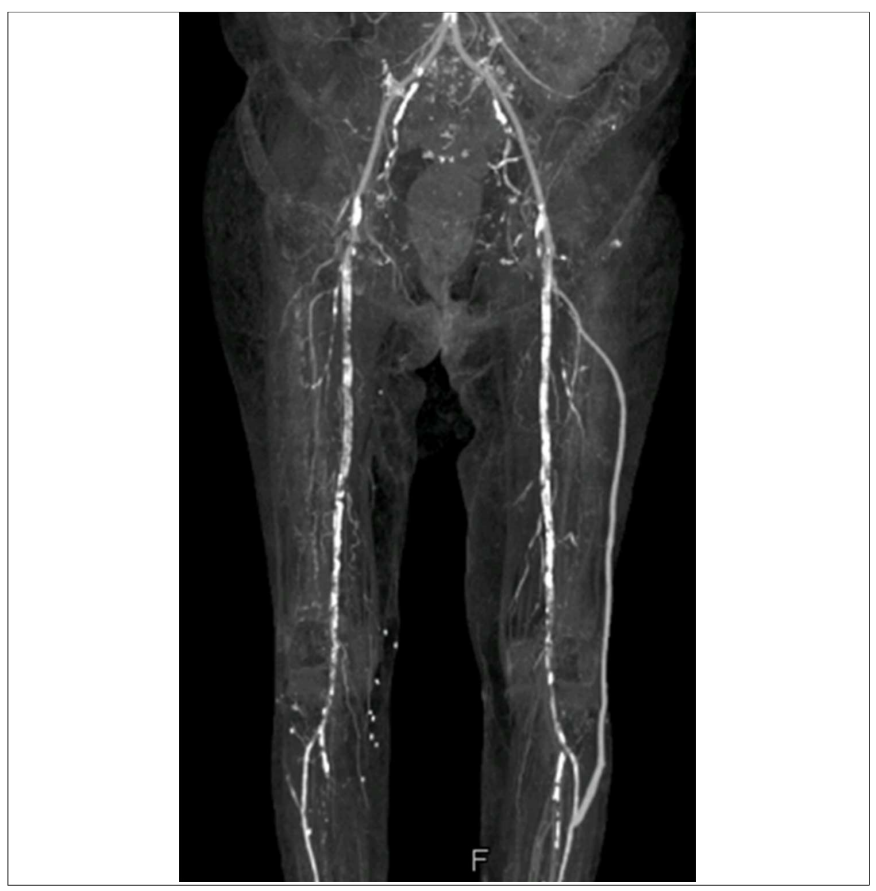

Figure 1: Computed tomographic angiogram showing patent left femoroanterior tibial vein bypass. The right bypass has occluded at 3 months but patient had viable leg with no symptoms.

the intended operative field. The disease was much more on left femoral bifurcation. Femoral endarterectomy was done. Ipsilateral GSV was used in reversed fashion; and it was also dissected by skip incisions avoiding the drained abscess site. She had smooth recovery from the procedure. Foot became warm and pink and her pain settled. She was walking after being bed bound for 4 months. On follow-up visit at 3 months of initial operation, she was asymptomatic but right graft had no pulsation. CT angiography (CTA) showed occluded right graft (Figure 1). As she was not having any complaints, no intervention was offered.

Lateral subcutaneous route is practical for scarred groins, active groin infection or thigh infection. Hoballah et al. first described lateral approach of ATA with subcutaneous graft tunnelling with primary patency of $16 \%$ and secondary patency of $86 \%$ at 1 year. They used this route for patients having medial infection, scarring from previous surgery, limited vein length, prior local radiotherapy and high-risk groins. ${ }^{5}$ Higher re-inter-ventions in these patients were due to advanced form of disease and limited quality of veins available. Our thought process was that a wound dehiscence on medial side can expose underlying graft, if placed on that side. This danger can potentially be avoided by extra-anatomical routing the graft away. In conventional route, the graft is deep and is not palpable compared to lateral subcutaneous route where the graft is easily palpable. Physicians and even patients themselves can monitor for any decreased flow and detect developing stenosis earlier. 
The graft on right side remained patent for three months and salvaged that leg.

Extra-anatomical route using the lateral subcutaneous tunnel is feasible for patients needing revascularisation of ATA.

\section{CONFLICT OF INTEREST:}

Authors declared no conflict of interest.

\section{AUTHOR'S CONTRIBUTION:}

ZUR: Substantial contributions to the conception or design of the work; the acquisition, analysis, or interpretation of data for the work; drafting the work or revising it critically for important intellectual content; final approval of the version to be published; agreement to be accountable for all aspects of the work in ensuring that questions related to the accuracy or integrity of any part of the work are appropriately investigated and resolved.

\section{REFERENCES}

1. Adam DJ, Beard JD, Cleveland T. Bypass versus angioplasty in severe ischemia of the leg (BASIL): Multicenter, randomised controlled trial. Lancet 2005; 366:1925-34.
2. Kontopodis N, Kafetzakis A. Profunda femoral to anterior tibial artery bypass to treat critical limb ischemia in a patient with numerous prior arterial reconstructions. Eur $J$ Vasc Endovasc Surg 2016; 52:702.

3. Benzing C, Fellomor PT. Lateral approach to the distal femoral artery in femoro-anterior-tibial bypass surgery. Eur $\mathrm{J}$ Vasc Endovasc Surg 2016; 52:267.

4. Lee T, Ra HD, Park YJ, Park HS, Kim SJ. New routing alternative for proximal anterior tibial artery bypasses in patients with Buerger disease. J Vasc Surg 2011; 54:1839-41.

5. Hoballah JJ, Chalmers RT, Sharp WJ. Lateral approach to the popliteal and crural vessels for limb salvage. Cardiovasc Surg 1996; 4:165-8.

Zia Ur Rehman

Department of Surgery, The Aga Khan University Hospital, Karachi, Pakistan

Correspondence to: Dr. Zia Ur Rehman, Section of Vascular Surgery, Department of Surgery, The Aga Khan University Hospital, Karachi, Pakistan

E-mail: ziaur.rehman@aku.edu

Received: March 08, 2019; Revised: May 08, 2019; Accepted: May 18, 2019 